\title{
Reduced expression of serum miR-204 predicts poor prognosis of gastric cancer
}

\author{
X. Chen ${ }^{1,2}$, X.S. Liu ${ }^{1,3}$, H.Y. Liu ${ }^{4}$, Y.Y. Lu ${ }^{1,3}$ and Y. Li ${ }^{5}$ \\ ${ }^{1}$ Qingdao University Medical College, Qingdao, Shandong, China \\ ${ }^{2}$ Department of Gastroenterology, Yantai Municipal Laiyang Central Hospital, \\ Yantai, Shandong, China \\ ${ }^{3}$ Department of Gastroenterology, The Affiliated Hospital of Qingdao University, \\ Qingdao, Shandong, China \\ ${ }^{4}$ Department of Infection Diseases, The Affiliated Hospital of Qingdao University, \\ Qingdao, Shandong, China \\ ${ }^{5}$ Department of Gastroenterology, Qingdao Municipal Hospital, Qingdao, \\ Shandong, China \\ Corresponding author: X.S. Liu \\ E-mail: xishuangliuqdu@163.com \\ Genet. Mol. Res. 15 (2): gmr.15027702 \\ Received September 22, 2015 \\ Accepted December 28, 2015 \\ Published April 25, 2016 \\ DOI http://dx.doi.org/10.4238/gmr.15027702
}

\begin{abstract}
Deregulation of microRNAs (miRNAs) is implicated in the initiation and progression of gastric cancer (GC). Previous studies have demonstrated that miR-204 was downregulated in GC tissues. However, its expression profile in serum samples and its potential for clinical value remain unknown. Real-time PCR was performed to evaluate the expression level of serum miR-204 in patients with GC. The association between serum miR-204 expression level and the clinical outcome of GC was then investigated. Our results showed that the expression of miR-204 in serum samples from GC patients was significantly lower than that in the healthy controls $(\mathrm{P}<0.01)$. Serum miR-204 expression level of GC patients was significantly upregulated after receiving surgical resection $(\mathrm{P}<0.01)$. In addition, serum miR204 was associated with lymph node metastasis $(\mathrm{P}=0.016)$, tumor
\end{abstract}


differentiation $(\mathrm{P}=0.001)$, and TNM stage $(\mathrm{P}=0.005)$. GC patients with low serum miR-204 expression had shorter overall survival than those with high serum miR-204 expression $(\mathrm{P}=0.004)$. Multivariate analysis revealed that serum miR-204 expression level was an independent risk factor for this malignant disease $(\mathrm{HR}=3.629,95 \% \mathrm{CI}=2.828-8.146$, $\mathrm{P}=0.015$ ). In conclusion, our findings indicate that serum miR-204 may be employed as a novel biomarker for monitoring the treatment response and predicting the prognosis of GC.

Key words: Gastric cancer; miR-204; Serum; Prognosis

\section{INTRODUCTION}

Gastric cancer (GC) is a heterogeneous, multi-factorial disease and the second most frequently diagnosed cancer (Jemal et al., 2006; Rahman et al., 2014). Despite a sharp decline in incidence and advances in surgical techniques, GC remains the second most common cause of cancer (Han et al., 2003). One of the major reasons for the poor clinical outcome of GC is that most patients are at an advanced clinical stage at the time of diagnosis (Dicken et al., 2005). Unfortunately, biomarkers with high sensitivity and specificity for GC are still lacking. Thus, identifying novel biomarkers for early detection and prediction of the prognosis of GC is important.

MicroRNAs (miRNAs) are a class of small, highly conservative, non-coding RNAs that mediate gene expression through complimentary binding with the 3 '-untranslated regions (UTRs) of target genes (He and Hannon, 2004). Abundant studies have shown that miRNAs regulate a variety of cellular processes, such as cell proliferation, migration, and differentiation (Bueno et al., 2008). Aberrant expression of miRNAs is closely correlated with many pathological processes, including human cancer (Dalmay and Edwards, 2006; Nana-Sinkam and Croce, 2011). Zhang et al. (2015b) showed that miR-153 was downregulated in GC tissues and that low expression of miR-153 was correlated with poor prognostic features. In addition, forced expression of miR-153 suppressed the migration and invasion capacity of GC cell lines and vice versa, indicating that miR-153 functions as a tumor suppressor in GC. Xu et al. (2015) reported that miR-374a was overexpressed in human GC cell lines and tissues. Inhibition of miR-374a was able to suppress GC cell proliferation, migration, and invasion in vitro and retarded tumor growth in vivo, suggesting that miR-374a may promote the tumorigenesis of GC.

Dysregulation of miR-204 is a common feature in a number of cancers, such as breast cancer, acute myeloid leukemia, and prostate cancer (Butrym et al., 2015; Wang et al., 2015; Todorova et al., 2016). Although miR-204 has been found to be downregulated in GC tissues (Zhang et al., 2015a), its expression profile in serum samples and its potential for clinical use are unknown. Therefore, the purpose of the current study was to investigate the clinical significance of serum miR-204 for GC.

\section{MATERIAL AND METHODS}

\section{Study population}

This study was approved by the Research Ethics Committee of the Affiliated Hospital of Qingdao University, and written informed consent was obtained from all participants, 
including 115 patients with GC and 40 healthy volunteers. GC patients were recruited from the Department of Gastroenterology, The Affiliated Hospital of Qingdao University; they were diagnosed with GC based on histopathological evaluation. No previous local or systemic treatment had been conducted on these GC patients before the first-time serum sample collection. Clinical data for the GC patients are summarized in Table 1.

\section{Clinical samples and real-time PCR}

Blood samples were collected from patients with GC or healthy donors. Serum was separated by centrifugation at $1200 \mathrm{~g}$ at $4^{\circ} \mathrm{C}$ for $15 \mathrm{~min}$ and then frozen at $-80^{\circ} \mathrm{C}$ prior to total RNA isolation. The miRNeasy Serum/Plasma Kit (Qiagen, Valencia, CA, USA) was used to isolate RNA from serum samples according to the manufacturer instructions. Briefly, $20 \mu \mathrm{L}$ total RNA sample was converted into cDNA using miScript-II-RT-Kit (Qiagen, Germany). Real-time PCR was then performed with the Applied Biosystems Prism 7500 Real-Time PCR System (Applied Biosystems, Foster City, CA, USA). Reactions were performed in triplicate, and miRNA relative expression was calculated using a $2^{-\Delta \Delta C t}$ method. RNU6 was used as an endogenous control.

\section{Statistical analysis}

Expression levels of serum miR-204 were compared between patients with GC and healthy volunteers using the Mann-Whitney U-test. The chi-square test was used to evaluate the association between serum miR-204 expression and clinical features of GC. An overall survival (OS) curve was plotted using the Kaplan-Meier method, and the association between serum miR-204 expression and OS in GC patients was analyzed using a log rank test. A Cox proportional hazard model was employed for multivariate analysis. All statistical analyses were performed using the SPSS version 22.0 for Windows software (SPSS, Inc., Chicago, $\mathrm{IL}$, USA). Data are reported as means $\pm \mathrm{SD}$, and differences were considered statistically significant when $\mathrm{P}<0.05$.

\section{RESULTS}

\section{Expression level of serum miR-204 in GC}

The results of the real-time PCR showed that the expression level of miR-204 in the serum samples from GC patients was significantly lower than that in the healthy controls $(\mathrm{P}<0.01$; Figure 1). Moreover, the serum miR-204 expression level in GC patients was significantly upregulated after receiving treatments $(\mathrm{P}<0.01$; Figure 2$)$, indicating that serum miR-204 might be employed to monitor treatment response.

\section{Association between serum miR-204 expression and clinicopathological parameters of GC}

The median fold-change in serum miR-204 was used as a cutoff value to divide all $115 \mathrm{GC}$ patients into two groups: the low-expression group $(\mathrm{N}=61)$ and the high-expression group $(\mathrm{N}=54)$. Serum miR-204 expression was associated with lymph node metastasis $(\mathrm{P}=$ 
0.016), tumor differentiation ( $\mathrm{P}=0.001)$, and tumor, node and metastasis (TNM) stage $(\mathrm{P}=$ $0.005)$. It was not correlated, however, with gender $(\mathrm{P}=0.755)$, age $(\mathrm{P}=0.861)$, tumor size $(\mathrm{P}$ $=0.207)$, or distant metastasis $(\mathrm{P}=0.095)($ Table 1$)$.

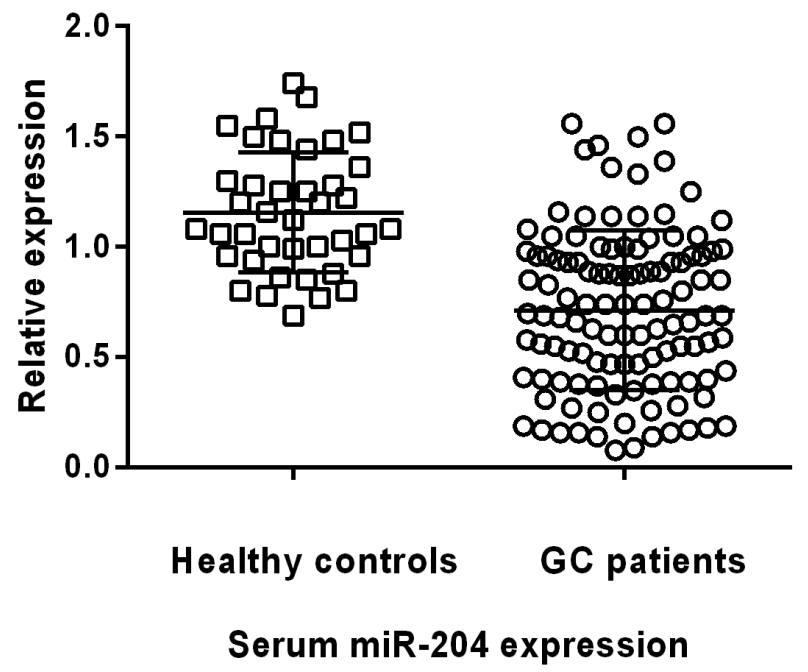

Figure 1. Expression level of serum miR-204 in gastric cancer (GC). The expression level of serum miR-204 was significantly downregulated in GC patients compared with that in healthy controls $(\mathrm{P}<0.01)$.

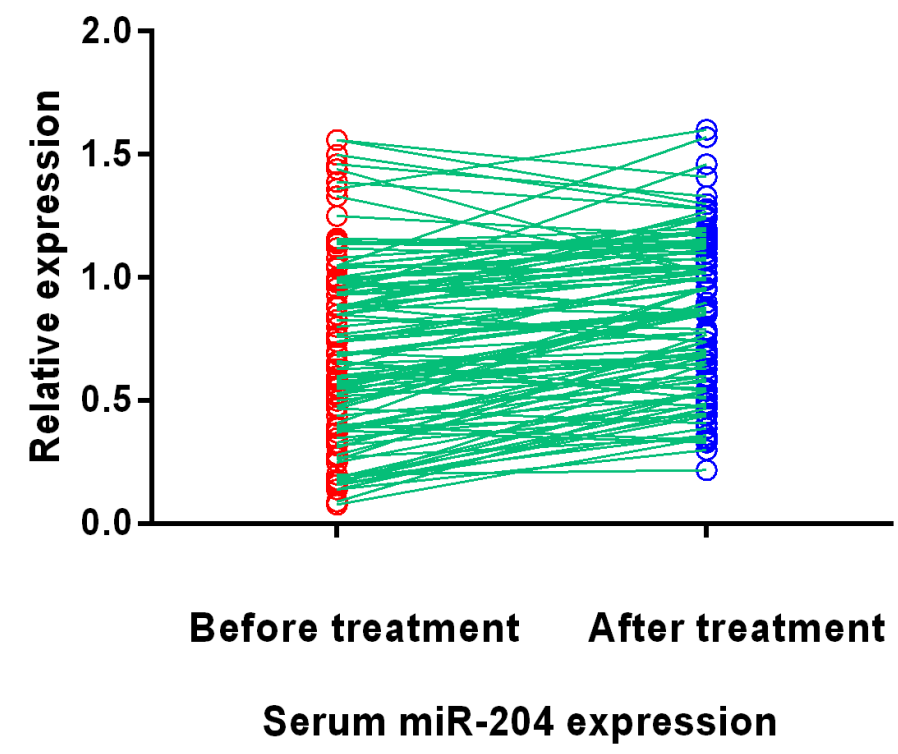

Figure 2. Serum miR-204 was upregulated in gastric cancer (GC) patients after therapy. Serum miR-204 expression level in GC patients was significantly increased after receiving treatments $(\mathrm{P}<0.01)$. 
Table 1. Correlations between serum miR-204 expression and clinicopathological features in GC patients.

\begin{tabular}{|c|c|c|c|c|}
\hline \multirow[t]{2}{*}{ Variables } & \multirow[t]{2}{*}{$\mathrm{N}$} & \multicolumn{2}{|c|}{ Serum miR-204 expression } & \multirow[t]{2}{*}{$\mathrm{P}$} \\
\hline & & Low & High & \\
\hline \multicolumn{5}{|l|}{ Gender } \\
\hline Male & 72 & 39 & 33 & 0.755 \\
\hline Female & 43 & 22 & 21 & \\
\hline \multicolumn{5}{|l|}{ Age (years) } \\
\hline$<60$ & 48 & 25 & 23 & 0.861 \\
\hline$\geq 60$ & 67 & 36 & 31 & \\
\hline \multicolumn{5}{|l|}{ Tumor size $(\mathrm{cm})$} \\
\hline$<5$ & 40 & 18 & 22 & 0.207 \\
\hline$\geq 5$ & 75 & 43 & 32 & \\
\hline \multicolumn{5}{|c|}{ Lymph node metastasis } \\
\hline No & 63 & 27 & 36 & 0.016 \\
\hline Yes & 52 & 34 & 18 & \\
\hline \multicolumn{5}{|l|}{ Distant metastasis } \\
\hline No & 71 & 19 & 25 & 0.095 \\
\hline Yes & 44 & 42 & 29 & \\
\hline \multicolumn{5}{|c|}{ Tumor differentiation } \\
\hline Well + Moderate & 56 & 21 & 35 & 0.001 \\
\hline Poor & 59 & 40 & 19 & \\
\hline \multicolumn{5}{|l|}{ TNM } \\
\hline I-II & 46 & 17 & 29 & 0.005 \\
\hline III-IV & 69 & 44 & 25 & \\
\hline
\end{tabular}

\section{Reduced serum miR-204 expression was associated with poor prognosis in GC patients}

Our survival analysis showed that GC patients with low serum miR-204 expression exhibited shorter overall survival than those with high serum miR-204 expression. The 5 -year OS rate in the low-expression group was $34.43 \%$, compared with $57.41 \%$ in the highexpression group $(\mathrm{P}=0.004$; Figure 3$)$.

Multivariate analysis revealed that tumor differentiation (hazard ratio $(\mathrm{HR})=4.206$, $95 \%$ confidence interval $(\mathrm{CI})=3.058-9.633, \mathrm{P}=0.008)$, $\mathrm{TNM}$ stage $(\mathrm{HR}=3.482,95 \% \mathrm{CI}=$ 2.674-7.410, $\mathrm{P}=0.021)$, and serum miR-204 expression $(\mathrm{HR}=3.629,95 \% \mathrm{CI}=2.828-8.146$, $\mathrm{P}=0.015$ ) were independent risk factors for GC (Table 2).

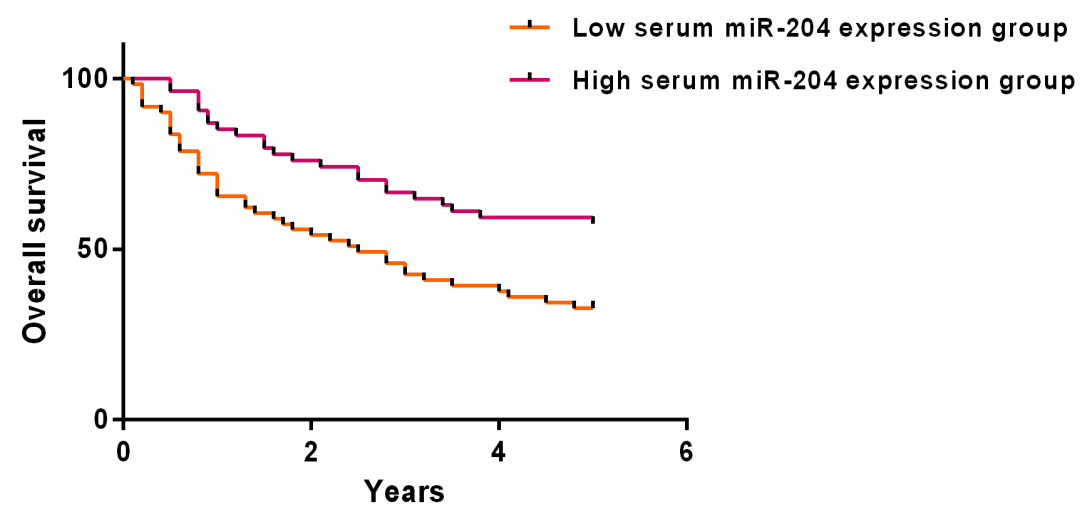

Figure 3. Association between serum miR-204 and overall survival in GC patients. The GC patients in the low serum miR-204 expression group had a significantly poorer overall survival than those in the high serum miR-204 expression group $(\mathrm{P}=0.004)$. 
Table 2. Multivariate analysis of overall survival in GC.

\begin{tabular}{l|c|c}
\hline Variables & \multicolumn{2}{|c}{ Overal survival } \\
\hline Lymph node metastasis & HR $(95 \% \mathrm{CI})$ & $\mathrm{P}$ \\
\hline Tumor differentiation & $1.806(0.981-2.893)$ & 0.053 \\
\hline TNM stage & $4.206(3.058-9.633)$ & 0.008 \\
\hline Serum miR-204 expression & $3.482(2.674-7.410)$ & 0.021 \\
\hline
\end{tabular}

\section{DISCUSSION}

The detection of biomarkers in serum samples provides several advantages over tissue biopsy. First, it is less invasive and more convenient. Serum biomarkers also may help clinicians classify GC patients into different subgroups before surgery resection and then provide reasonable treatment options. Lastly, biomarkers allow for the monitoring of treatment responses of patients in real-time, which may significantly improve treatment outcomes. In this study, our data showed that serum miR-204 expression was reduced in GC patients compared with healthy volunteers. In addition, miR-204 was significantly upregulated after the GC patients received surgical treatments. Overexpression of serum miR-204 was associated with advanced clinical stage and poor OS for GC. Moreover, serum miR-204 was an independent risk factor for this malignant disease.

Our findings indicate that miR-204 may function as a tumor suppressor in the development of GC, which is consistent with previous studies documenting the role of miR204 in GC. The expression level of miR-204 has been reported to be downregulated in GC tissues and cell lines by different research groups (Zhang et al., 2013, 2015a). Zhang et al. (2015a) showed that miR-204-5p suppression could promote the proliferative capacity of GC cell lines, whereas forced expression of miR-204 inhibited this capacity. In addition, USP47 and RAB22A were identified as direct functional targets of miR-204-5p in GC (Zhang et al., 2015a). An inverse relationship was found between miR-204 expression and SIRT1 expression in GC tissues, and overexpression of miR-204 was found to reduce GC cell invasion, anoikis resistance, and epithelial-mesenchymal transition by targeting SIRT-1 at the post-transcriptional level. Therefore, miR-204 is believed to play a crucial role in regulating the metastasis of GC (Zhang et al., 2013). Ezrin expression is also upregulated in GC tissues and cell lines, and overexpression of Ezrin has been correlated with poor prognosis of GC. As Ezrin is critical to Ras activation and is predicted to be a target of miR-204, this indicates that miR-204 downregulation may be a novel mechanism for aberrant Ras activation in gastric tumorigenesis (Lam et al., 2011). Further, miR-204 may target Bcl-2 expression and increase the responsiveness of GC cells to 5-fluorouracil and oxaliplatin treatment, indicating that miR-204 might be a therapeutic target for improving the prognosis of GC (Sacconi et al., 2012). Wang et al. (2014) compared differentially expressed miRNAs between Helicobacter pylori-related gastritis and gastric intestinal metaplasia lesions. MiR-204 was downregulated in GC precancerous lesions and may be closely correlated with the progression of GC. The expression of miR-204 has been found to be enhanced in $H$. pylori-negative GC compared with $H$. pylori-positive GC, suggesting that distinct GC pathogenesis mechanisms may exist in H. pylori-positive and H. pylori-negative GC and that miR-204 expression may be correlated with $H$. pylori infection (Chang et al., 2015).

As miRNAs may regulate many downstream target genes, it is common for a specific miRNA to act as an oncogene in one type of cancer while functioning as a tumor 
suppressor in another. However, to the best of our knowledge, miR-204 has been shown to inhibit tumorigenesis in all types of cancers investigated thus far. The expression level of miR-204-5p was significantly downregulated in glioma tissues compared with normal brain tissues. Moreover, restoration of miR-204-5p expression resulted in suppression of tumor cell behaviors and improvement in overall host survival, suggesting that targeting miR-204-5p may be a practical and effective strategy for treating gliomas (Xia et al., 2015). Yin et al. (2014) revealed that miR-204-5p was frequently downregulated in colorectal cancer tissues and that low miR-204-5p expression was associated with poor clinical outcome. In addition, ectopic miR-204-5p expression not only inhibited the proliferation, migration, and invasion capacity of colorectal cancer cells, but also enhanced tumor sensitivity to chemotherapy by suppressing RAB22A expression. Similarly, Qiu et al. (2013) reported that the relative expression of miR204 was reduced in human intrahepatic cholangiocarcinoma (ICC) as well as ICC cell lines and that reduced miR-204 expression was correlated with lymph node metastasis. Overexpression of miR-204 inhibited ICC cell migration, invasion, and the epithelial-mesenchymal transition process by targeting Slug directly. Although miR-204 plays a tumor suppressive role in many different types of cancers, its downstream targeted genes may be distinct in each type. Further investigations are needed to confirm the clinical significance of miR-204 in GC and elucidate its role in the carcinogenesis of GC.

\section{CONCLUSIONS}

In conclusion, we revealed that serum miR-204 was downregulated in patients with GC. A low level of serum miR-204 was associated with tumor progression and poor clinical outcome of GC. These findings indicate that serum miR-204 may be a good candidate for the prognosis prediction in GC patients.

\section{Conflicts of interest}

The authors declare no conflict of interest.

\section{ACKNOWLEDGMENTS}

We want to acknowledge the patients and the healthy volunteers participating in the study, as well as the medical personnel who help collect the clinical samples.

\section{REFERENCES}

Bueno MJ, Pérez de Castro I and Malumbres M (2008). Control of cell proliferation pathways by microRNAs. Cell Cycle 7: 3143-3148. http://dx.doi.org/10.4161/cc.7.20.6833

Butrym A, Rybka J, Baczyńska D, Tukiendorf A, et al. (2015). Low expression of microRNA-204 (miR-204) is associated with poor clinical outcome of acute myeloid leukemia (AML) patients. J. Exp. Clin. Cancer Res. 34: 68. http://dx.doi. org/10.1186/s13046-015-0184-Z

Chang H, Kim N, Park JH, Nam RH, et al. (2015). Different microRNA expression levels in gastric cancer depending on Helicobacter pylori infection. Gut Liver 9: 188-196. http://dx.doi.org/10.5009/gnl13371

Dalmay T and Edwards DR (2006). MicroRNAs and the hallmarks of cancer. Oncogene 25: 6170-6175. http://dx.doi. org/10.1038/sj.onc.1209911

Dicken BJ, Bigam DL, Cass C, Mackey JR, et al. (2005). Gastric adenocarcinoma: review and considerations for future directions. Ann. Surg. 241: 27-39. 
Han JY, Son H, Lee WC and Choi BG (2003). The correlation between gastric cancer screening method and the clinicopathologic features of gastric cancer. Med. Oncol. 20: 265-269. http://dx.doi.org/10.1385/MO:20:3:265

He L and Hannon GJ (2004). MicroRNAs: small RNAs with a big role in gene regulation. Nat. Rev. Genet. 5: 522-531. http://dx.doi.org/10.1038/nrg1379

Jemal A, Siegel R, Ward E, Murray T, et al. (2006). Cancer statistics, 2006. CA Cancer J. Clin. 56: 106-130. http://dx.doi. org/10.3322/canjclin.56.2.106

Lam EK, Wang X, Shin VY, Zhang S, et al. (2011). A microRNA contribution to aberrant Ras activation in gastric cancer. Am. J. Transl. Res. 3: 209-218.

Nana-Sinkam SP and Croce CM (2011). Non-coding RNAs in cancer initiation and progression and as novel biomarkers. Mol. Oncol. 5: 483-491. http://dx.doi.org/10.1016/j.molonc.2011.10.003

Qiu YH, Wei YP, Shen NJ, Wang ZC, et al. (2013). miR-204 inhibits epithelial to mesenchymal transition by targeting slug in intrahepatic cholangiocarcinoma cells. Cell. Physiol. Biochem. 32: 1331-1341. http://dx.doi.org/10.1159/000354531

Rahman R, Asombang AW and Ibdah JA (2014). Characteristics of gastric cancer in Asia. World J. Gastroenterol. 20: 4483-4490. http://dx.doi.org/10.3748/wjg.v20.i16.4483

Sacconi A, Biagioni F, Canu V, Mori F, et al. (2012). miR-204 targets Bcl-2 expression and enhances responsiveness of gastric cancer. Cell Death Dis. 3: e423. http://dx.doi.org/10.1038/cddis.2012.160

Todorova K, Metodiev MV, Metodieva G, Zasheva D, et al. (2016). miR-204 is dysregulated in metastatic prostate cancer in vitro. Mol. Carcinog. 55: 131-147. http://dx.doi.org/10.1002/mc.22263

Wang X, Qiu W, Zhang G, Xu S, et al. (2015). MicroRNA-204 targets JAK2 in breast cancer and induces cell apoptosis through the STAT3/BCl-2/survivin pathway. Int. J. Clin. Exp. Pathol. 8: 5017-5025.

Wang XW, Wu Y, Wang D and Qin ZF (2014). MicroRNA network analysis identifies key microRNAs and genes associated with precancerous lesions of gastric cancer. Genet. Mol. Res. 13: 8695-8703. http://dx.doi.org/10.4238/2014. October. 27.10

Xia Z, Liu F, Zhang J and Liu L (2015). Decreased expression of miRNA-204-5p contributes to glioma progression and promotes glioma cell growth, migration and invasion. PLoS One 10: e0132399. http://dx.doi.org/10.1371/journal. pone. 0132399

Xu X, Wang W, Su N, Zhu X, et al. (2015). miR-374a promotes cell proliferation, migration and invasion by targeting SRCIN1 in gastric cancer. FEBS Lett. 589: 407-413. http://dx.doi.org/10.1016/j.febslet.2014.12.027

Yin Y, Zhang B, Wang W, Fei B, et al. (2014). miR-204-5p inhibits proliferation and invasion and enhances chemotherapeutic sensitivity of colorectal cancer cells by downregulating RAB22A. Clin. Cancer Res. 20: 6187-6199. http://dx.doi. org/10.1158/1078-0432.CCR-14-1030

Zhang B, Yin Y, Hu Y, Zhang J, et al. (2015a). MicroRNA-204-5p inhibits gastric cancer cell proliferation by downregulating USP47 and RAB22A. Med. Oncol. 32: 331. http://dx.doi.org/10.1007/s12032-014-0331-y

Zhang L, Wang X and Chen P (2013). MiR-204 down regulates SIRT1 and reverts SIRT1-induced epithelialmesenchymal transition, anoikis resistance and invasion in gastric cancer cells. BMC Cancer 13: 290. http://dx.doi. org/10.1186/1471-2407-13-290

Zhang Z, Sun J, Bai Z, Li H, et al. (2015b). MicroRNA-153 acts as a prognostic marker in gastric cancer and its role in cell migration and invasion. Onco Targets Ther. 8: 357-364. 